\title{
Transarterial Chemoembolization of Hepatocellular Carcinoma Using Radiopaque Drug-Eluting Embolics: Impact of Embolic Density and Residual Tumor Perfusion on Tumor Recurrence and Survival
}

\author{
Christer Ruff $^{1,2}$ (D) Christoph Artzner ${ }^{2} \cdot$ Roland Syha $^{3} \cdot$ Ulrich Grosse $^{4} \cdot$ \\ Rüdiger Hoffmann ${ }^{2} \cdot$ Michael Bitzer $^{5} \cdot$ Sasan Partovi $^{6} \cdot$ Marius Horger $^{2}$. \\ Konstantin Nikolaou ${ }^{2} \cdot$ Gerd Grözinger $^{2}$
}

Received: 15 December 2020/ Accepted: 24 April 2021/Published online: 21 May 2021

(C) The Author(s) 2021

\begin{abstract}
Purpose To evaluate the value of dual-phase parenchymal blood volume (PBV) C-arm mounted cone-beam-CT (CBCT) to enable assessment of radiopaque, doxorubicinloaded drug-eluting embolics (rDEE) based on the visual degree of embolization, embolic density and residual tumor perfusion as early predictors for tumor recurrence after transarterial chemoembolization (TACE) of hepatocellular carcinoma (HCC).

Material and Methods Thirty patients (50 HCCs) were prospectively enrolled, underwent cross-sectional imaging before and after TACE using 100-300 $\mu \mathrm{m}$ rDEE and had regular follow-up examinations. Directly before and after
\end{abstract}

Supplementary Information The online version contains supplementary material available at https://doi.org/10.1007/s00270021-02858-6.

Christer Ruff

christer.ruff@uni-tuebingen.de

1 Department of Diagnostic and Interventional Neuroradiology, University Hospital Tuebingen, Hoppe-Seyler-Strasse 3, 72076 Tuebingen, Germany

2 Department of Diagnostic and Interventional Radiology, University Hospital Tuebingen, Hoppe-Seyler-Strasse 3, 72076 Tuebingen, Germany

3 Department of Diagnostic and Interventional Radiology, Prosper Hospital, Muehlenstraße 27, 45659 Recklinghausen, Germany

4 Department of Radiology, Spital Thurgau AG, Waldeggstraße 8A, 8501 Frauenfeld, Switzerland

5 Department for Internal Medicine I, University Hospital Tuebingen, Otfried-Mueller-Strasse 10, 72076 Tuebingen, Germany

6 Section of Interventional Radiology, Imaging Institute, Cleveland Clinic Main Campus, Cleveland, OH, USA the TACE procedure, PBV-CBCT was acquired. The response was evaluated and compared to visual degree of embolization (DE) and embolic density (ED) of rDEE deposits, as well as the presence of residual tumor perfusion (RTP) derived from PBV-CBCT. Outcome was assessed by mid-term tumor response applying mRECIST and patient survival after 12 months.

Results RTP was detected in 16 HCCs and correlated negatively with $\mathrm{DE}(p=.03 *)$ and $\operatorname{ED}(p=.0009 *)$. The absence of RTP significantly improved lesion-based midterm response rates regarding complete response (CR, $30 / 34(88 \%)$ vs $2 / 16(12.5 \%), p=.0002 *)$, lesion-based complete response rate was $75 \%(21 / 28)$ for $\mathrm{DE} \geq 50 \%$ vs. $50 \%(11 / 22)$ for $\mathrm{DE}<50 \%(p=.08)$ and $82 \%(27 / 33)$ for $\mathrm{ED} \geq 2$ vs. $29 \%$ for $\left.\mathrm{ED}<2(5 / 17), p=.005^{*}\right)$. Thirteen patients were treated with re-TACE within 12 months, 11 of which had shown RTP. 12-month survival rate was $93 \%$. Conclusion Residual tumor perfusions as assessed by PBV-CBCT during rDEE-TACE proved to be the best parameter to predict mid-term response.

"Level of Evidence: Level 3"

Keywords Hepatocellular carcinoma (HCC) .

Transarterial chemoembolization (TACE) .

Radiopaque drug-eluting embolics (rDEE) · C-arm mounted cone-beam CT (CBCT) 


\section{Introduction}

Transarterial chemoembolization (TACE) is an established treatment for intermediate-stage hepatocellular carcinoma (HCC) or as a bridge to liver transplantation (LT) for earlystage HCC [1]. Recently developed radiopaque drug-eluting embolics (rDEE) (DC Bead LUMI ${ }^{\mathrm{TM}}$, Boston Scientific plc, Marlborough, MA, the USA) for TACE comprise radiopaque microspheres which enable direct visualization with conventional X-ray imaging [2]. This might aid in more precise delivery of rDEE including superior peri- and post-procedural visualization of their extent and distribution. C-Arm mounted cone-beam CT (CBCT) can be performed during the procedure in the angiography suite for immediate peri-interventional assessment of tumor extent and vascularization $[3,4]$. CBCT enhances the detection of tumor feeders and, therefore, gains an increasing importance for TACE guidance [3]. Furthermore, CBCT performed directly after TACE has demonstrated the ability to improve the detection of residual tumor perfusion (RTP) and thus helps to tailor individual treatment [3]. However, due to their inherent opacity, rDEE prohibits the proper visualization of residual tumor after TACE with a singlephase contrast-enhanced CBCT as embolics cannot be distinguished from arterial or parenchymal contrast staining. Therefore, a dual-phase CBCT is pursued with a nonenhanced and a contrast-enhanced phase. This allows the subtraction of the datasets and the calculation of parenchymal blood volume (PBV) and hence measurement of tumor perfusion [3].

An important question arising when using rDEE relates to the possible relationship between their accumulation in HCCs after TACE, the treatment response and, ultimately, patient-centered outcome like tumor recurrence and survival. This parameter already plays a role in predicting the outcome after cTACE with Lipiodol [5]. The implications of peri-procedural evaluation of PBV-CBCT for rDEEtreatment response and outcome have not been elucidated yet. In this prospective study, the extent and localization of rDEE deposit and density as well as RTP were evaluated and correlated with clinical outcomes like tumor response according to the modified Response Evaluation Criteria in Solid Tumors (mRECIST) after 3 months and patient survival at 12 months post- initial rDEE-TACE.

\section{Materials and Methods}

The local institutional ethics committee approved this single-center prospective study. Before enrollment, written informed consent was obtained from all participating subjects.

\section{Patient Population}

Between 06/2017 and 06/2019, 30 patients were prospectively enrolled. Detailed patient data are summarized in Table 1. The study included 25 men (mean age $69.4 \pm 8.4$ years, range 53-83 years) and five women (mean age $71.1 \pm 8.3$ years, range $68-83$ years) with a total number of 50 HCCs. 13 of these 30 enrolled patients were part of another, previously published study [6]. All patients were classified as stage A or B according to the Barcelona clinic liver cancer (BCLC) classification. The average number of tumor lesions per patient was $1.7 \pm 0.9$ [range 1-5]. The average lesion diameter was $31.2 \pm 20 \mathrm{~mm}$. Inclusion and exclusion criteria for TACE were in line with the CIRSE standards of practice guidelines including blood values contained therein [7]. Criteria were age $\geq 18$ years, single or oligo-focal HCC $(\leq 5$ HCCs), no previous treatment by embolization or ablation therapy, multidisciplinary tumor board decision for TACE treatment and imaging including multiphase CT and MRI, not older than 21 days prior to TACE treatment.

\section{Imaging}

All patients received pre-interventional cross-sectional imaging with multiphase CT (volume perfusion CT) including non-enhanced CT scans (SOMATOM Definition Flash, Siemens Healthineers, Forchheim, Germany) and GD-EOB-DTPA-enhanced (Primovist ${ }^{\circledR}$; Bayer Healthcare, Leverkusen, Germany) multiphase 3 T MRI examinations (Magnetom VIDA, Siemens Healthineers, Erlangen, Germany). Follow-up cross-sectional imaging examinations were performed every 3 months after TACE for a period of 12 months, including multiphase CT or MRI, according to a previously published protocol [6]. Technical details of CT acquisitions are given in Supplemental Table 1.

\section{Transarterial Chemoembolization}

All interventions were performed utilizing the same robotic angiographic suite (Artis Zeego Q, VE 40A, Siemens Healthineers, Forchheim, Germany). Femoral access was used in all cases. Aortography was performed in cases where extrahepatic tumor supply or variant anatomy was suspected. 2.7 or 2.4 Fr microcatheters (Progreat; Terumo, Leuven, Belgium) were used in a $4 \mathrm{Fr}$ catheter for superselective hepatic angiography and embolization using radiopaque polyvinyl alcohol (PVA) embolic microspheres (DC Bead LUMI ${ }^{\mathrm{TM}}$; 100-300 $\mu \mathrm{m}$; Boston Scientific plc, Marlborough, MA, the USA) loaded with $50 \mathrm{mg}$ doxorubicin. rDEE were reconstituted in pure contrast media (Ultravist 300®, Bayer Healthcare, Leverkusen, Germany) to keep the microspheres in suspension. The administration 
Table 1 Baseline characteristics of the 30 enrolled subjects with a total of $50 \mathrm{HCC}$ lesions. BCLC = Barcelona clinic liver cancer classification

\begin{tabular}{|c|c|c|c|c|c|c|}
\hline Patient & Age & Sex & BCLC classification & Number of lesions & Liver segments & Tumor characteristics \\
\hline 1 & 66 & $\mathrm{~m}$ & B & 2 & $\begin{array}{l}\text { VIII } \\
\text { IV }\end{array}$ & $\begin{array}{l}\text { encapsulated } \\
\text { encapsulated }\end{array}$ \\
\hline 2 & 64 & $\mathrm{~m}$ & $\mathrm{~B}$ & 2 & $\begin{array}{l}\mathrm{II} / \mathrm{III} \\
\mathrm{VIII}\end{array}$ & $\begin{array}{l}\text { infiltrative with portal vein invasion } \\
\text { encapsulated }\end{array}$ \\
\hline 3 & 72 & $\mathrm{~m}$ & A & 1 & V/VIII & encapsulated \\
\hline 4 & 65 & $\mathrm{~m}$ & A & 1 & II & infiltrative \\
\hline 5 & 64 & $\mathrm{~m}$ & A & 1 & II/III & encapsulated \\
\hline 6 & 83 & $\mathrm{~m}$ & B & 1 & II/III & encapsulated \\
\hline 7 & 58 & $\mathrm{~m}$ & B & 1 & III/IV & encapsulated \\
\hline 8 & 79 & $\mathrm{~m}$ & A & 1 & $\mathrm{~V} / \mathrm{VI}$ & encapsulated \\
\hline 9 & 83 & $\mathrm{~m}$ & B & 1 & VI/VII/VIII & encapsulated \\
\hline 10 & 62 & $\mathrm{~m}$ & B & 5 & $\begin{array}{l}\mathrm{V} \\
\mathrm{IV}\end{array}$ & $\begin{array}{l}\text { encapsulated } \\
\text { encapsulated }\end{array}$ \\
\hline 11 & 53 & $\mathrm{~m}$ & A & 2 & $\begin{array}{l}\mathrm{V} \\
\mathrm{IV}\end{array}$ & $\begin{array}{l}\text { encapsulated } \\
\text { encapsulated }\end{array}$ \\
\hline 12 & 62 & $\mathrm{~m}$ & B & 1 & IV/VIII & encapsulated \\
\hline 13 & 67 & $\mathrm{~m}$ & A & 1 & IV & encapsulated \\
\hline 14 & 71 & $\mathrm{~m}$ & B & 2 & $\begin{array}{l}\text { III } \\
\text { IV }\end{array}$ & $\begin{array}{l}\text { encapsulated } \\
\text { encapsulated }\end{array}$ \\
\hline 15 & 67 & $\mathrm{~m}$ & B & 3 & $\begin{array}{l}\text { VI } \\
\text { VI } \\
\text { VIII }\end{array}$ & $\begin{array}{l}\text { encapsulated } \\
\text { encapsulated } \\
\text { encapsulated }\end{array}$ \\
\hline 16 & 70 & $\mathrm{~m}$ & B & 2 & $\begin{array}{l}\mathrm{II} / \mathrm{IV} \\
\mathrm{VI}\end{array}$ & $\begin{array}{l}\text { encapsulated } \\
\text { encapsulated }\end{array}$ \\
\hline 17 & 54 & $\mathrm{~m}$ & A & 1 & III & encapsulated \\
\hline 18 & 80 & $\mathrm{~m}$ & A & 1 & II & encapsulated \\
\hline 19 & 65 & $\mathrm{~m}$ & A & 2 & $\begin{array}{l}\text { VI } \\
\text { VIII }\end{array}$ & $\begin{array}{l}\text { encapsulated } \\
\text { encapsulated }\end{array}$ \\
\hline 20 & 80 & $\mathrm{~m}$ & A & 1 & IV & encapsulated \\
\hline 21 & 76 & $\mathrm{~m}$ & A & 3 & $\begin{array}{l}\text { IV/VIII } \\
\text { VII } \\
\text { VII/VIII }\end{array}$ & $\begin{array}{l}\text { encapsulated } \\
\text { encapsulated } \\
\text { encapsulated }\end{array}$ \\
\hline 22 & 76 & $\mathrm{~m}$ & B & 1 & VI & encapsulated \\
\hline 23 & 81 & $\mathrm{~m}$ & B & 1 & VIII & encapsulated \\
\hline 24 & 69 & $\mathrm{~m}$ & B & 2 & $\begin{array}{l}\text { II } \\
\text { IV }\end{array}$ & $\begin{array}{l}\text { encapsulated } \\
\text { encapsulated }\end{array}$ \\
\hline 25 & 69 & $\mathrm{~m}$ & B & 2 & $\begin{array}{l}\text { VII/VIII } \\
\text { VIII }\end{array}$ & $\begin{array}{l}\text { encapsulated } \\
\text { encapsulated }\end{array}$ \\
\hline 26 & 58 & $\mathrm{w}$ & A & 1 & VII/VIIII & encapsulated \\
\hline 27 & 76 & $\mathrm{w}$ & B & 3 & $\begin{array}{l}\text { I } \\
\text { II/III } \\
\text { VI }\end{array}$ & $\begin{array}{l}\text { encapsulated } \\
\text { encapsulated } \\
\text { encapsulated }\end{array}$ \\
\hline 28 & 71 & $\mathrm{w}$ & A & 1 & VII/VIII & Infiltrative \\
\hline 29 & 83 & w & B & 2 & $\begin{array}{l}\text { II } \\
\text { VII }\end{array}$ & $\begin{array}{l}\text { encapsulated } \\
\text { encapsulated }\end{array}$ \\
\hline 30 & 68 & w & B & 2 & $\begin{array}{l}\text { II/IV } \\
\text { VIII }\end{array}$ & $\begin{array}{l}\text { encapsulated } \\
\text { encapsulated }\end{array}$ \\
\hline
\end{tabular}




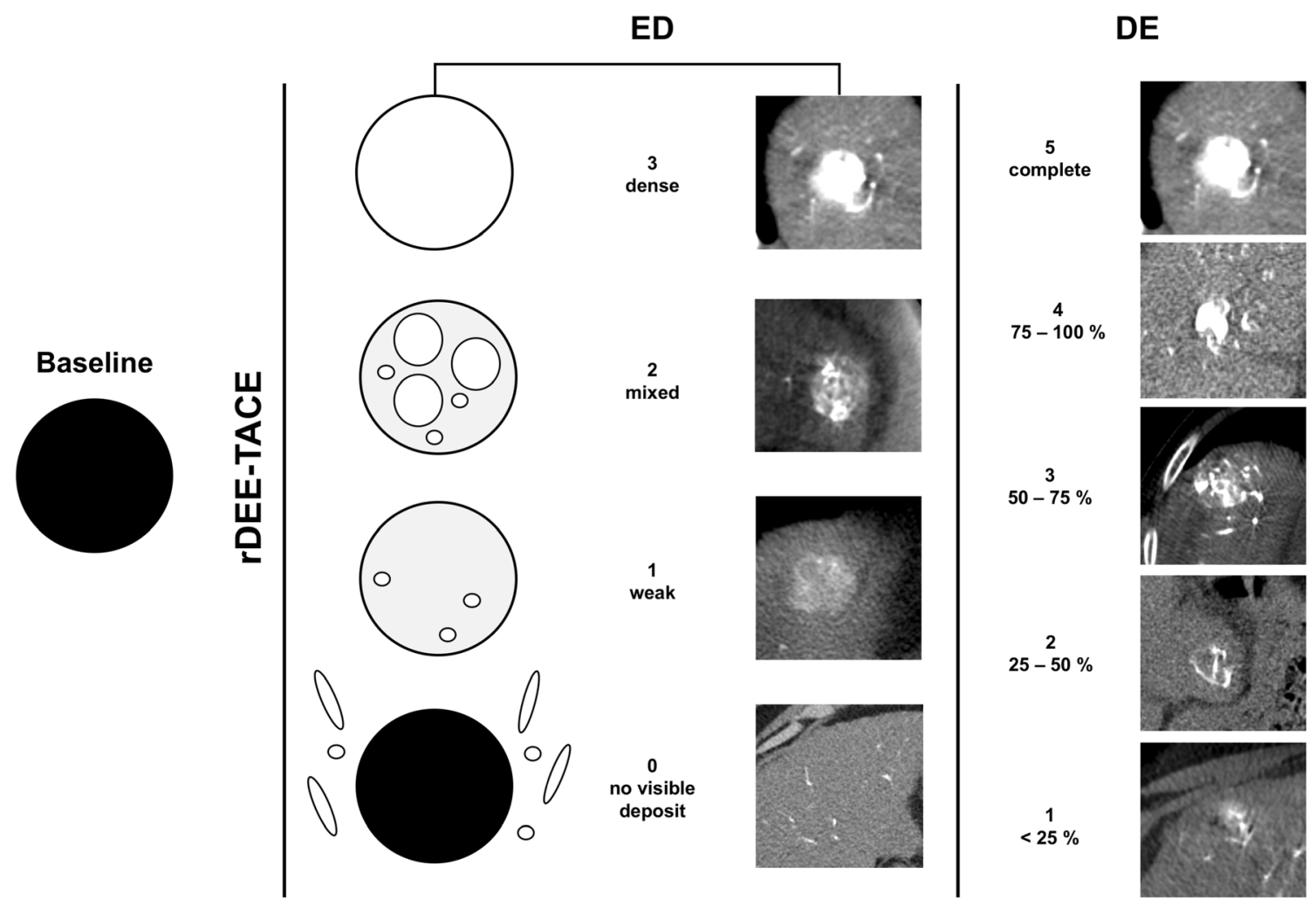

Fig. 1 Image examples for the classification of embolic density (ED) and visual degree of embolization (DE). ED and DE (\% of the tumor area in cross-sectional images filled with dense radiopaque embolics) of treated HCCs were assessed qualitatively using an ordinal 4-point
Likert scale for $\operatorname{ED}(3=$ dense; 2 = mixed; 1 = weak, 0 = not visible $)$ and 6-point Likert scale for DE ( $5=$ complete; $4=75-100 \%$; $3=50-75 \% ; 2=25-50 \% ; 1=<25 \%, 0=$ no visible deposit $)$

described previously, are given in Supplemental Table 1 $[3,8,9]$.

\section{Data Analysis}

\section{Dual-Phase Parenchymal Blood Volume C-Arm Mounted Cone-Beam-CT (PBV-CBCT)}

Two sets of PBV-CBCT images of the liver were acquired during the TACE procedure. The first dataset was acquired 5 min after hepatic arterial angiography before administering rDEE. The second dataset was acquired after near stasis and stop of the embolization to assess RTP. Each CBCT consisted of an unenhanced phase ("mask run") and a contrast-enhanced phase ("fill run"). Fill run and mask run were subtracted. A non-rigid registration algorithm was performed to mitigate motion-related differences between the two runs. The arterial input function value is calculated from an automated histogram analysis of the vessel tree and applied as a scaling factor to obtain the actual PBV map [12]. Technical details of image acquisition and postprocessing using an automatic reconstruction algorithm, as

\section{Evaluation of Tumor Size, Residual Tumor Perfusion, Embolic Density and Degree of Embolization}

In pre-interventional CBCT-PBV maps, maximum lesion diameter was recorded. All post-interventional non-enhanced runs were evaluated for visible contrast media and rDEE deposit. In the case of a present deposit, the maximum diameter of each deposit was measured. Density of rDEE embolization (embolic density, ED) and visual degree of embolization (DE, \% of the tumor area in crosssectional images filled with dense embolic material) of treated HCC lesions were assessed qualitatively using an 4-point Likert scale ( $3=$ dense; $2=$ mixed; 1 = weak, $0=$ not visible) for $\mathrm{ED}$ and a 6-point Likert scale for DE $(5=$ complete $; 4=75-100 \% ; 3=50-75 \% ; 2=25-$ $50 \% ; 1=<25 \%, 0=$ no visible deposit), Fig. 1 and Table 2. ED and DE were evaluated on a representative 
Table 2 Assessment of HCC lesions treated with rDEE-TACE and results of $50 \mathrm{HCC}$ lesions treated with rDEE-TACE according to visual degree of embolization (DE), embolic density (ED) and residual tumor perfusion (RTP)

\begin{tabular}{lcc}
\hline Likert scale & Meaning & number of HCC lesions \\
\hline \multicolumn{2}{l}{ Visual degree of embolization (DE) } & \\
5 & Complete & 7 \\
4 & $75-100 \%$ & 11 \\
3 & $50-75 \%$ & 10 \\
2 & $25-50 \%$ & 10 \\
1 & $<25 \%$ & 6 \\
0 & no visible deposit & 6 \\
Embolic density (ED) & 18 \\
3 & Dense & 15 \\
2 & Mixed & 11 \\
1 & Weak & 6 \\
0 & not visible & 16 \\
Residual arterial enhancement / tumor perfusion (RTP) \\
Yes & 34 \\
No & & \\
\hline
\end{tabular}

An ordinal 4-point Likert scale for ED $(3=$ dense; $2=$ mixed; $1=$ weak, $0=$ not visible) and a 6-point Likert scale for DE (5 = complete; $\quad 4=75-100 \% ; \quad 3=50-75 \% ; \quad 2=25-50 \%$; $1=<25 \%, 0=$ no visible deposit) was used for assessment of rDEE. Mean DE was $2.7 \pm 1.6[1-3,3-5]$, and the mean ED was $1.9 \pm 1.0[1,2,2,3]$

slice position. Based on the distribution of DE and ED of the HCCs, a categorization of the $\mathrm{DE} \geq 50 \%$ vs. $<50 \%$ and ED of $\geq 2$ vs. $<2$ was established for further evaluation (Fig. 2, Table 2). On PBV maps, evaluation of residual tumor perfusion of the entire tumor or parts of it was assessed and maximum perfused tumor areas were measured. Two authors (both with 8 and 12 years of experience in cross-sectional imaging and with 4 and 5 years of experience in interventional radiology, respectively) reviewed peri-procedural and follow-up CT, CBCT and MR images in a consensus reading. The readers were blinded to the initial TACE procedure and clinical outcomes including results of mid-term tumor response and 12-month survival.

\section{Assessment of Mid-Term Treatment Response}

Baseline and 3-month follow-up cross-sectional imaging were evaluated applying mRECIST. Patients were classified as progressive disease (PD, an increase of at least $20 \%$ in diameter of the viable lesion), stable disease (SD, any case that does not qualify for either partial response or PD), partial response (PR, at least a $30 \%$ decrease in the diameter of the viable lesion), or complete response (CR, the disappearance of any intralesional arterial enhancement). The objective response rate (ORR) was defined as the proportion of patients with CR or PR. Evaluation of tumor recurrence after TACE was performed based on each individual lesion and patient-based.

\section{Survival Analysis at 12 Months}

Survival analysis was performed at 12 months after the initial TACE. The number of orthotopic liver transplantations (OLT) and the number of repeated TACE procedures were recorded.

\section{Statistical Analysis}

Statistical analysis was performed using JMP 14.2 (SAS Institute Inc., Cary, NC, the USA) and SPSS (release 26 for Windows; SPSS, Chicago, IL, the USA). Median values and interquartile ranges were calculated for the applied ordinal Likert scales. Pearson's Chi-square test for independence was applied for correlation of DE, ED and RTP. For comparison of all qualitative image parameters of individual patients, Friedman-ANOVA analysis was conducted. If a significant difference was detected, the paired Wilcoxon-signed-rank test was subsequently performed. Due to multiple testing, Bonferroni corrected $\mathrm{p}$ values were determined. A corrected $\mathrm{p}$ value of equal or less than 0.05 was considered statistically significant.

\section{Results}

\section{Evaluation of Tumor Size, Residual Tumor Perfusion, Embolic Density and Degree of Embolization}

Three of 50 HCCs (6\%) showed a diffuse, infiltrative growth pattern, whereas 47 of $50 \mathrm{HCCs}$ (94\%) were encapsulated. The mean size of HCCs in pre-interventional CBCT was $31.2 \pm 20 \mathrm{~mm}$. Complete devascularization was achieved in 34 of 50 HCCs $(68 \%)$. RTP could be detected in 16/50 HCCs (32\%). Of the 16 HCCs with RTP, $5 \mathrm{HCCs}$ had a DE $\geq 50 \%$ and $\mathrm{ED} \geq 2$, whereas $11 \mathrm{HCCs}$ had a $\mathrm{DE}<50 \%$ and ED $<2$. RTP was associated with $\mathrm{DE}<50 \%(11 / 16(69 \%), \rho=0.0309 *)$ and $\mathrm{ED}<2(11 /$ $16(69 \%), \rho=0.0009 *)$, Fig. 3 and 4 . There was no significant difference of the size of HCCs with RTP $(30.5 \pm 11.9 \mathrm{~mm}) \quad$ compared to HCCs without detectable RTP $(30.8 \pm 21.3 \mathrm{~mm}), p=0.96$. The size of detected RTP was $16.1 \pm 8.7 \mathrm{~mm}$ in CBCT. The largest size of RTP was $26.2 \mathrm{~mm}$. 
Fig. 2 Distribution of HCC lesions according to embolic density (ED) and visual degree of embolization (DE). ED and $\mathrm{DE}(\%$ of the tumor area in cross-sectional images filled with dense radiopaque embolics) of treated HCCs were assessed qualitatively using an ordinal 4-point Likert scale for ED $(3$ = dense; 2 = mixed; $1=$ weak, $0=$ not visible) and 6-point Likert scale for DE $(5=$ complete; $4=75-100 \%$; $3=50-75 \% ; 2=25-50 \%$; $1=\langle 25 \%, 0=$ no visible deposit). Mean ED was $1.9 \pm$ $1.0[2,0-3]$ and mean DE was $2.7 \pm 1.6[3,0-5]$

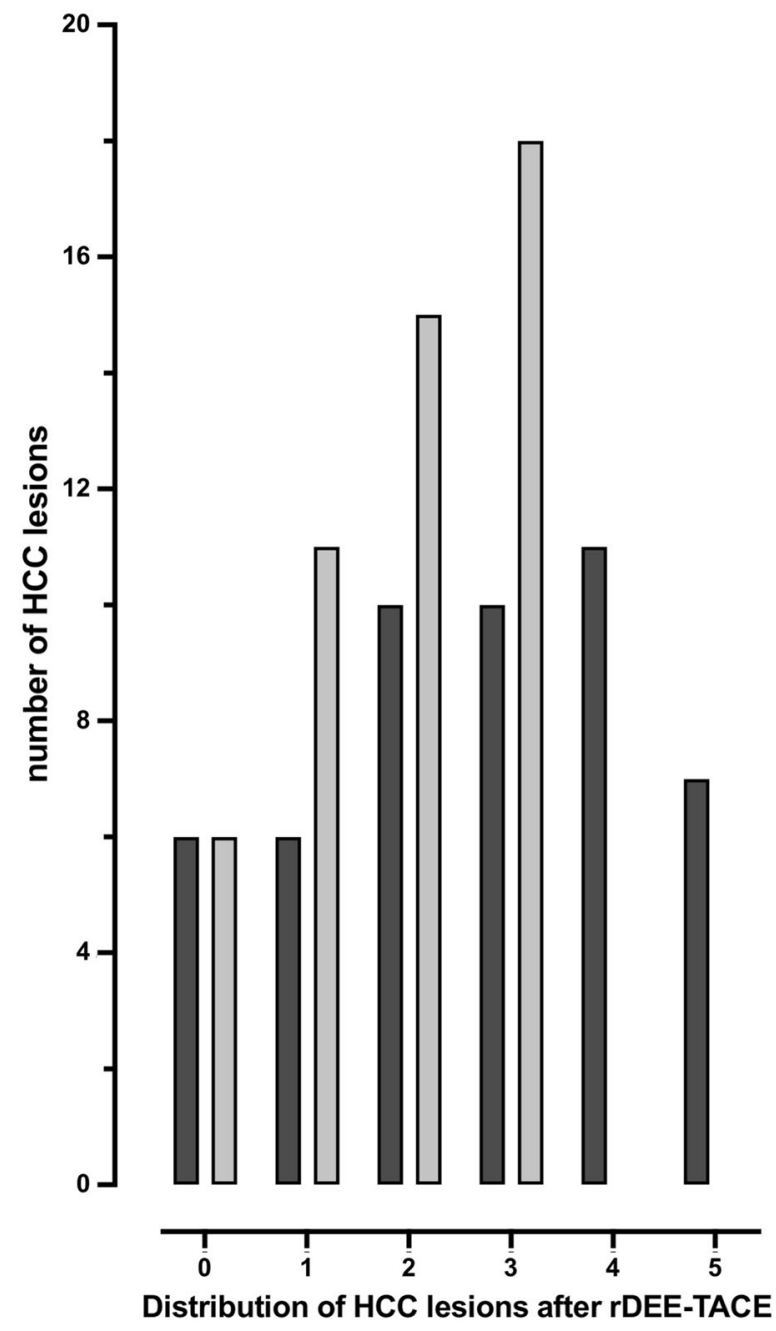

significantly different between these two groups $(32 / 33$ (97\%) vs. $10 / 17(58 \%), p=0.0062 *)$.

\section{Survival Analysis at 12 Months}

Overall survival at 12 months was $93 \%$ (28/30 patients). At 12 months, 9/30 patients had received orthotopic liver transplantation $\left(7.4 \pm 3.4\right.$ months after the $1^{\text {st }}$ TACE). 13/30 patients had received a second TACE procedure within the first 12 months of follow-up. Of these, 11/13 patients had at least one HCC lesion which had shown RTP in the initial post-TACE PBV-CBCT.

\section{Discussion}

In this study, the value of post-interventional PBV-CBCT could be demonstrated using radiopaque embolics. It enables the assessment of rDEE deposition parameters like embolic density and degree of embolization together with 

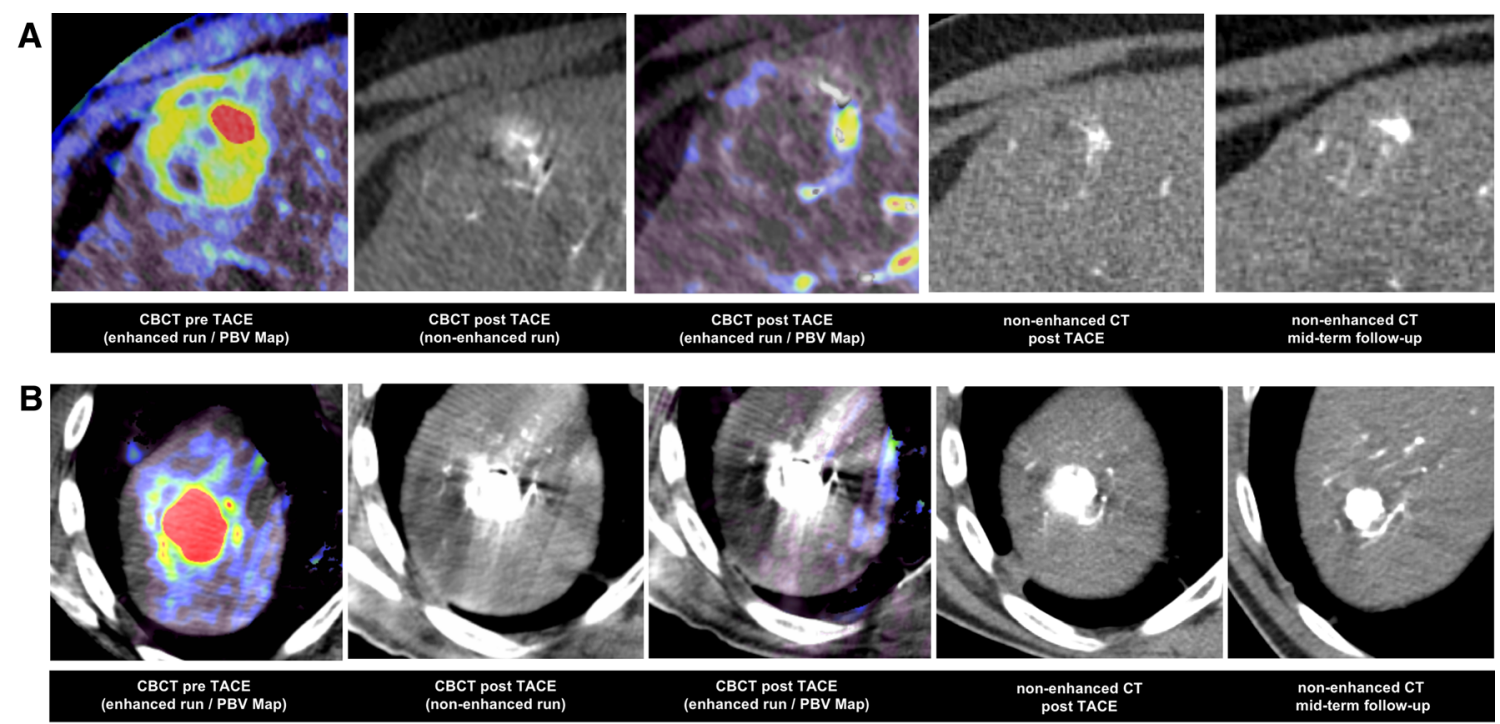

CBCT post TACE
$($ enhanced run / PBV Ma

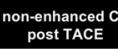

non-enhanced CT
mid-term follow-up
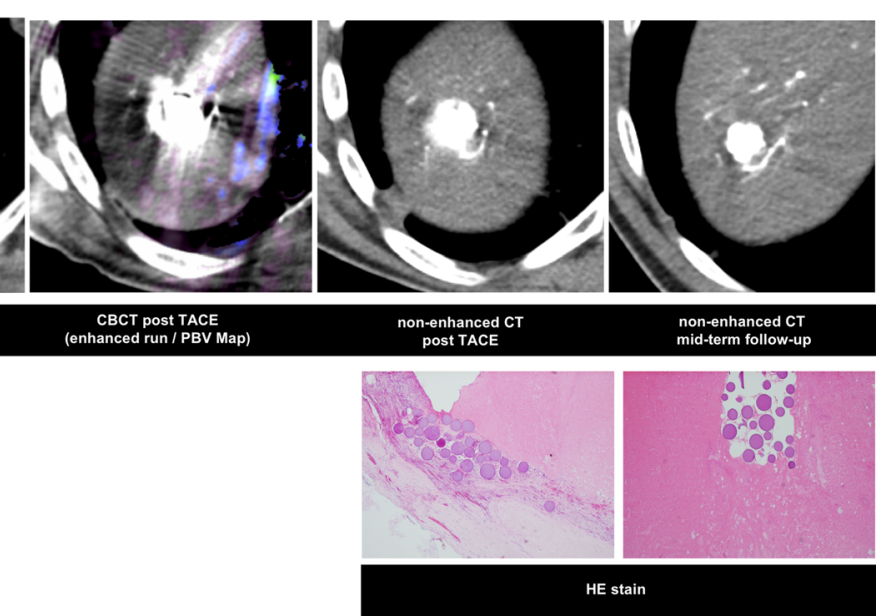

Fig. 3 Patient examples with assessment of residual tumor perfusion, embolic density (ED) and degree of embolization (DE) of two patients with HCC lesions post-TACE using radiopaque drug-eluting embolic microspheres (rDEE). A A 54-year-old patient with DE of segment III HCC lesion of $<25 \%$ and weak ED ( 1 = weak). PBV map shows residual tumor perfusion (RTP) post-procedurally. B A 58-year-old patient with complete embolization (100\%) and a high

the detection of residual tumor perfusion in treated HCCs. Residual tumor perfusion has proved to be a robust parameter regarding prediction of mRECIST and is influenced by embolic density as well as degree of embolization.

Recently developed rDEE are supposed to improve visibility and lesion targeting [10]. This prospective study, therefore, evaluated CBCT imaging biomarkers in the periinterventional management of HCCs treated with rDEETACE. The aim was to establish early predictors for midterm tumor response. Non-enhanced CBCT images were evaluated to assess the extent and the visibility of rDEE, whereas PBV-CBCT was used to directly measure residual tumor perfusion after the embolization procedures [3]. Perfusion-based imaging has been shown to accurately assess hypovascularized HCCs as well as the achieved level of tumor devascularization [3, 11-17]. Syha et al. could show that detection of residual tumor perfusion in PBV-CBCT imaging of treated HCCs with drug-eluting embolics was associated with a worse mid-term response. In this study using rDEE, the most robust imaging density ( $3=$ dense) of segment VIII HCC lesion post-TACE. In PBV maps, no RTP can be delineated. However, beam hardening artifacts caused by high DC Bead LUMI ${ }^{\mathrm{TM}}$ concentration in target lesion is slightly limiting interpretation. The patient received orthotopic liver transplantation 4 months after TACE. Histologic specimen (Hematoxylin and eosin (HE) staining) showed densely packed microspheres within the vessels of the tumor bed surrounded by tumor necrosis

biomarker also was the absence of residual tumor perfusion with regard to the prediction of mid-term response. The rate of residual tumor perfusion (32\%) is in line with previous studies $[3,18]$. If possible, detection of residual tumor perfusion should lead to immediate re-treatment. However, immediate treatment with further embolization is not always possible due to the occurence of post-embolization syndrome or impaired liver function with and increasing risk of liver damage.

In this study, rDEE were reconstituted in pure contrast media in all procedures. This is necessary to avoid the immediate sinking of the relatively heavy radiopaque particles. Because of that, the contrast staining within the tumor is certainly iodine-containing contrast medium combined with rDEE. However, this is a constant among all procedures and a dual-phase CBCT enables the discrimination of residual tumor perfusion and staining in the tumor bed. As described in the literature, radiopaque embolics tend to form clots at high concentrations in a model of a phantom study [6]. Furthermore, non-enhanced CT imaging one day after TACE procedure and on mid- 

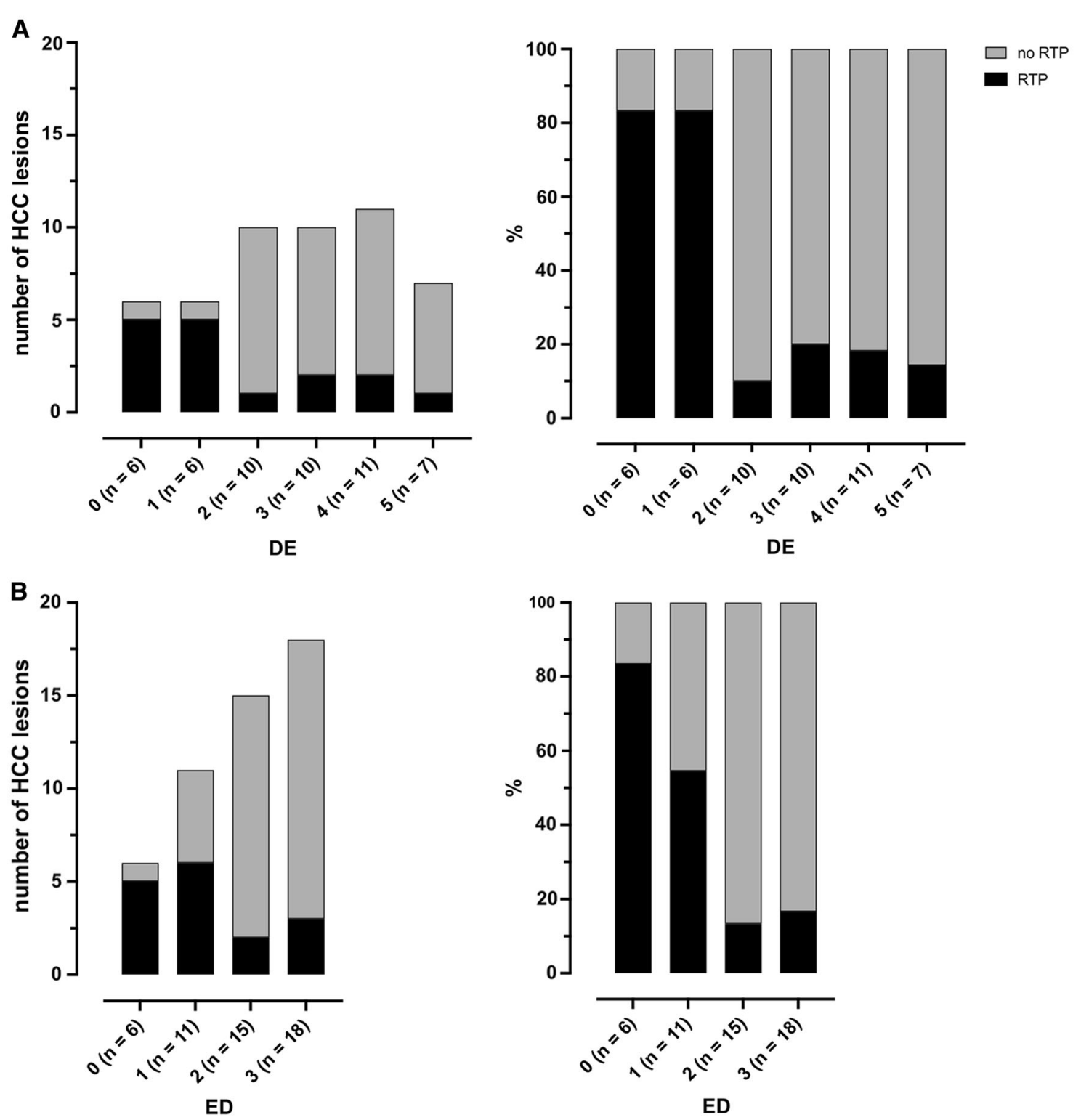

Fig. 4 Relationship of residual tumor perfusion (RTP) to degree of embolization (DE, A) and embolic density (ED, B). Data given in absolute and percentage terms 

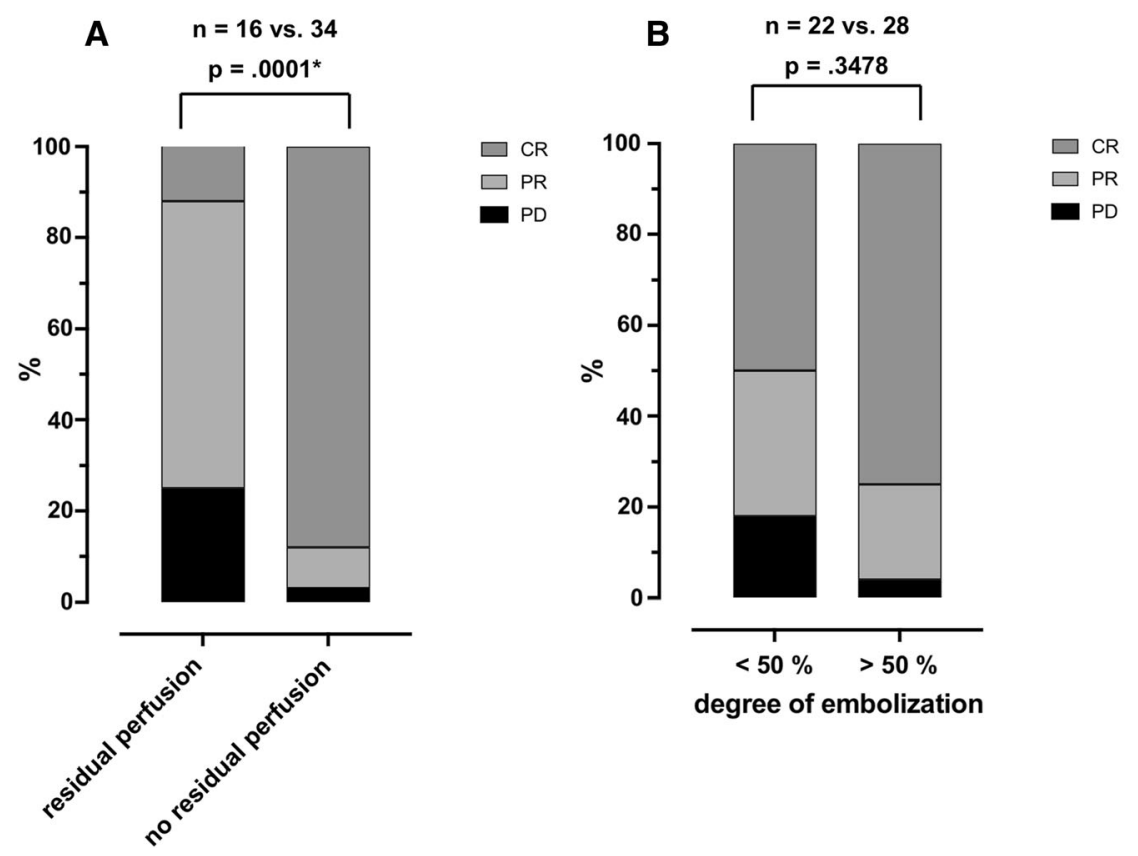

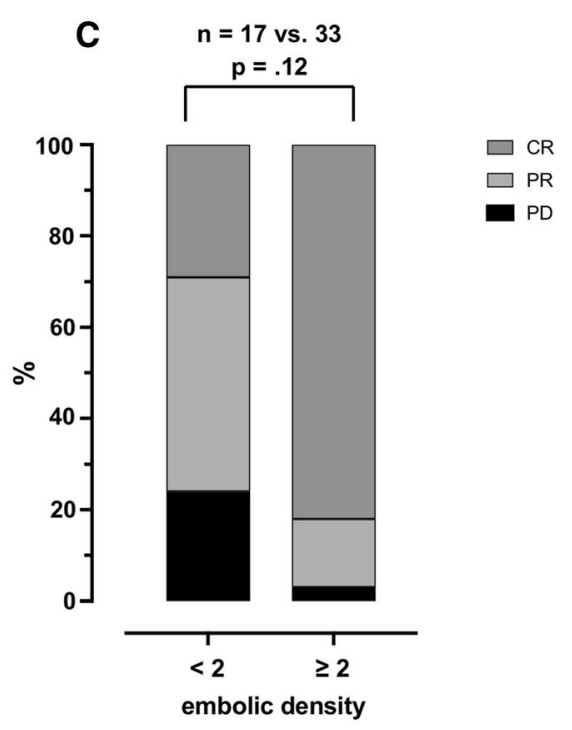

Fig. 5 Mid-term response in relation to residual tumor perfusion (RTP, A), visual degree of embolization (DE, B $) \geq /<50 \%$ and embolic density $(\mathrm{ED}, \mathbf{C})</ \geq 2$ in relationship to complete response $(\mathrm{CR})$, partial response (PR) and progressive disease (PD) based on
mRECIST (modified Response Evaluation Criteria in Solid Tumors) criteria. HCC lesions without RTP show superior mid-term tumor response three months post-rDEE-TACE
Table 3 Results of 50 HCC lesions treated with $\mathrm{rDEE}$ -

TACE according to visual degree of embolization (DE), embolic density (ED) and residual tumor perfusion (RTP) as well as evaluation based on mRECIST (Modified Response Evaluation Criteria in Solid Tumors) criteria.

ORR $=$ objective response rate, $\mathrm{CR}=$ complete response,

$\mathrm{PR}=$ partial response,

$\mathrm{PD}=$ progressive disease

\begin{tabular}{|c|c|c|c|c|}
\hline \multicolumn{5}{|c|}{ mRECIST (lesion-based) } \\
\hline & ORR & $\mathrm{CR}$ & PR & PD \\
\hline \multicolumn{5}{|c|}{ Visual degree of embolization (DE) } \\
\hline$<50$ & $18 / 22(82 \%)$ & $11 / 22(50 \%)$ & $7 / 22(31 \%)$ & $4 / 22(18 \%)$ \\
\hline$>50$ & $27 / 28(96 \%)$ & $21 / 28(75 \%)$ & $6 / 28(21 \%)$ & $1 / 28(5 \%)$ \\
\hline$<50$ vs. $>50$ & .08 & .08 & .78 & .215 \\
\hline \multicolumn{5}{|c|}{ Embolic density (ED) } \\
\hline$<2$ & $10 / 17(58 \%)$ & $5 / 17(29 \%)$ & $8 / 17(47 \%)$ & $4 / 17(24 \%)$ \\
\hline$\geq 2$ & $32 / 33(97 \%)$ & $27 / 33(82 \%)$ & $5 / 33(15 \%)$ & $1 / 33(3 \%)$ \\
\hline$<2$ vs. $\geq 2$ & $.0062^{*}$ & $.005 *$ & .4097 & .215 \\
\hline \multicolumn{5}{|c|}{ Residual tumor perfusion (RTP) } \\
\hline Yes & $12 / 16(75 \%)$ & $2 / 16(12.5 \%)$ & $4 / 16(25 \%)$ & $10 / 16(62.5 \%)$ \\
\hline No & $33 / 34(97 \%)$ & $30 / 34(88 \%)$ & $1 / 34(3 \%)$ & $3 / 34(9 \%)$ \\
\hline yes vs. no & $.019 *$ & $.0002 *$ & .0674 & .2150 \\
\hline
\end{tabular}


term follow-up imaging shows a very similar distribution of the embolics compared to the time of post-interventional CBCT. Low embolic density and degree of embolization were specific findings that were closely linked to the presence of residual tumor perfusion. As such, these parameters may play a complementary role as outcome predictors, even when no dual-phase CBCT with the ability to create PBV maps is available. In these cases, the deposit of rDEE can also be visualized in a separate non-enhanced CT scan one day after rDEE-TACE. A decreased embolic density was significantly associated with an unfavorable mid-term outcome in the form of a reduced rate of complete response. These parameters were already established to predict the outcome after conventional TACE (cTACE) with Lipiodol [5]. In cases with residual tumor perfusion and low embolic density, short re-treatment intervals versus the combination with other local or systemic therapeutic strategies (e.g., radiofrequency ablation) are strategies to improve the individual patient outcome. This is in line with a previous study showing that shorter retreatment intervals (e.g., six versus 12 weeks) could improve the outcome in cases with residual tumor perfusion after the first TACE session [8].

rDEE distribution and density might pave the way for a "rDEE dosimetry." This is supported by the results of Sharma et al., who demonstrated that rDEE concentration could be correlated with CT attenuation. Subsequently, the degree of radiopacity post-TACE can be correlated with the delivered drug concentration to the HCC lesions (19). Although rDEE dosimetry was not directly applied in this study, it was demonstrated that embolic density and degree of embolization are linked to the established imaging biomarker of residual tumor perfusion and, thus, might help to estimate the tumor response.

The study has several limitations. The study population consists of a comparably small sample size and the followup period was limited to 12 months. Nevertheless, the study results point to a potential path toward a more individualized oncological strategy.

\section{Conclusion}

In conclusion, the results of this study demonstrate the value of post-interventional PBV-CBCT to enable assessment of rDEE deposition parameters like embolic density and visual degree of density together with the detection of residual tumor perfusion in treated HCCs. Residual tumor perfusion has proved to be a robust parameter regarding mid-term response according to mRECIST.

Funding Open Access funding enabled and organized by Projekt DEAL. This study was not supported by any funding.

\section{Declarations}

Conflict of interest The authors declare that they have no conflict of interest.:

Consent for Publication Consent for publication was obtained for every individual person's data included in the study.

Ethical Approval All procedures performed in studies involving human participants were in accordance with the ethical standards of the institutional and / or national research committee and with the 1964 Helsinki declaration and its later amendments or comparable ethical standards.

Informed Consent Informed consent was obtained from all individual participants included in the study.

Open Access This article is licensed under a Creative Commons Attribution 4.0 International License, which permits use, sharing, adaptation, distribution and reproduction in any medium or format, as long as you give appropriate credit to the original author(s) and the source, provide a link to the Creative Commons licence, and indicate if changes were made. The images or other third party material in this article are included in the article's Creative Commons licence, unless indicated otherwise in a credit line to the material. If material is not included in the article's Creative Commons licence and your intended use is not permitted by statutory regulation or exceeds the permitted use, you will need to obtain permission directly from the copyright holder. To view a copy of this licence, visit http://creativecommons. org/licenses/by/4.0/.

\section{References}

1. Lencioni R, Petruzzi P, Crocetti L. Chemoembolization of hepatocellular carcinoma. Semin Intervent Radiol. 2013;30(1):3-11.

2. Dreher MR, Sharma KV, Woods DL, Reddy G, Tang Y, Pritchard $\mathrm{WF}$, et al. Radiopaque drug-eluting beads for transcatheter embolotherapy experimental study of drug penetration and coverage in swine. Journal of vascular and interventional radiology JVIR. 2012;23(2):257-64 e4.

3. Syha R, Grozinger G, Grosse U, Maurer M, Zender L, Horger M, et al. Parenchymal blood volume assessed by c-arm-based computed tomography in immediate posttreatment evaluation of drug-eluting bead transarterial chemoembolization in hepatocellular carcinoma. Invest Radiol. 2016;51(2):121-6.

4. Orth RC, Wallace MJ, Kuo MD. Technology Assessment Committee of the Society of Interventional R. C-arm cone-beam CT: general principles and technical considerations for use in interventional radiology. Journal of vascular and interventional radiology: JVIR. 2008;19(6):814-20.

5. Dioguardi Burgio M, Sartoris R, Libotean C, Zappa M, Sibert A, Vilgrain $\mathrm{V}$, et al. Lipiodol retention pattern after TACE for HCC is a predictor for local progression in lesions with complete response. Cancer Imaging. 2019;19(1):75.

6. Ruff C, Grozinger G, Syha R, Elser S, Partovi S, Bitzer M, et al. Transarterial chemoembolization of hepatocellular carcinoma using radiopaque drug-eluting embolics: how to pursue periprocedural cross-sectional imaging? Journal of vascular and interventional radiology: JVIR. 2019;30(3):380-9 e4.

7. Basile A, Carrafiello G, Ierardi AM, Tsetis D, Brountzos E. Quality-improvement guidelines for hepatic transarterial 
chemoembolization. Cardiovasc Intervent Radiol. 2012;35(4):765-74.

8. Syha R, Ketelsen D, Heller S, Schmehl J, Mangold S, Heuschmid $\mathrm{M}$, et al. Hepatocellular carcinoma: initial tumour response after short-term and long-interval chemoembolization with drug-eluting beads using modified RECIST. Eur J Gastroenterol Hepatol. 2012;24(11):1325-32.

9. Zitzelsberger T, Syha R, Grozinger G, Partovi S, Nikolaou K, Grosse U. Image quality of arterial phase and parenchymal blood volume (PBV) maps derived from C-arm computed tomography in the evaluation of transarterial chemoembolization. Cancer Imaging. 2018;18(1):16.

10. Ashrafi K, Tang Y, Britton H, Domenge O, Blino D, Bushby AJ, et al. Characterization of a novel intrinsically radiopaque Drugeluting Bead for image-guided therapy: DC Bead LUMI. J Control Release. 2017;250:36-47.

11. Chen G, Ma DQ, He W, Zhang BF, Zhao LQ. Computed tomography perfusion in evaluating the therapeutic effect of transarterial chemoembolization for hepatocellular carcinoma. World J Gastroenterol. 2008;14(37):5738-43.

12. Choi SH, Chung JW, Kim HC, Baek JH, Park CM, Jun S, et al. The role of perfusion CT as a follow-up modality after transcatheter arterial chemoembolization: an experimental study in a rabbit model. Invest Radiol. 2010;45(7):427-36.

13. Ippolito D, Bonaffini PA, Ratti L, Antolini L, Corso R, Fazio F, et al. Hepatocellular carcinoma treated with transarterial chemoembolization: dynamic perfusion-CT in the assessment of residual tumor. World J Gastroenterol. 2010;16(47):5993-6000.

14. Jiang T, Kambadakone A, Kulkarni NM, Zhu AX, Sahani DV. Monitoring response to antiangiogenic treatment and predicting outcomes in advanced hepatocellular carcinoma using image biomarkers, CT perfusion, tumor density, and tumor size (RECIST). Invest Radiol. 2012;47(1):11-7.

15. Kaufmann S, Schulze M, Spira D, Horger M. Comparison of volume perfusion computed tomography and contrast-enhanced ultrasound for assessment of therapeutic effect of transarterial chemoembolization in patients with hepatocellular carcinoma: a preliminary report. Acta Radiol. 2016;57(1):8-12.

16. Chandarana H, Block TK, Ream J, Mikheev A, Sigal SH, Otazo $\mathrm{R}$, et al. Estimating liver perfusion from free-breathing continuously acquired dynamic gadolinium-ethoxybenzyl-diethylenetriamine pentaacetic acid-enhanced acquisition with compressed sensing reconstruction. Invest Radiol. 2015;50(2):88-94.

17. Weiss J, Ruff C, Grosse U, Grozinger G, Horger M, Nikolaou K, et al. Assessment of hepatic perfusion using GRASP MRI: bringing liver MRI on a new level. Invest Radiol. 2019;54(12):737-43.

18. Ippolito D, Fior D, Bonaffini PA, Capraro C, Leni D, Corso R, et al. Quantitative evaluation of CT-perfusion map as indicator of tumor response to transarterial chemoembolization and radiofrequency ablation in HCC patients. Eur $\mathrm{J}$ Radiol. 2014;83(9):1665-71.

19. Sharma KV, Dreher MR, Tang Y, Pritchard W, Chiesa OA, Karanian J, et al. Development of "imageable" beads for transcatheter embolotherapy. J Vasc Interv Radiol. 2010;21(6):865-76.

Publisher's Note Springer Nature remains neutral with regard to jurisdictional claims in published maps and institutional affiliations. 